\title{
Usefulness of a Narrow-Band Reflectance Spectrophotometer in Evaluating Effects of Depigmenting Treatment
}

\author{
Kotaro Yoshimura, M.D., Kiyonori Harii, M.D., Yuji Masuda, B.S., Motoji Takahashi, Ph.D., Takao Aoyama, Ph.D., \\ and Tatsuji Iga, Ph.D.
}

Tokyo, Japan

\begin{abstract}
As a depigmenting treatment, combined topical applications of all-trans retinoic acid (atRA) aqueous gel and 5\% hydroquinone, $7 \%$ lactic acid ointment were used for Oriental patients with hyperpigmented skin lesions such as senile lentigines and nevus spilus. A narrow-band reflectance spectrophotometer and a tristimulus colorimeter were used to evaluate objectively the intensity of pigmentation and erythema at each clinical visit. $L^{*}, a^{*}$, and $b^{*}$ values measured with a tristimulus colorimeter (Chroma Meter CR-300) enabled the evaluation of erythema but not pigmentation. On the other hand, the melanin and hemoglobin values measured with a narrow-band reflectance spectrophotometer (Mexameter MX-16) expressed both erythema and pigmentation well. It was revealed that, in our bleaching protocol, the narrow-band reflectance spectrophotometer was quite useful for estimating accurately the intensity of pigmentation and erythema and determining the best time point for the cessation of atRA treatment.
\end{abstract}

Key words: Depigmenting treatment-Retinoic acid aqueous gel-Senile lentigines-Nevus spilus

Using our bleaching protocol with a high concentration of all-trans retinoic acid (atRA) aqueous gel in combination with hydroquinone and lactic acid, a remarkable improvement of various skin lesions with hyperpigmentation, such as senile lentigines [13], melasma, postinflammatory hyperpigmentation, and nevus spilus, was obtained with a short period of treatment [14]. However, during the treatment, side effects such as erythema were frequently observed after the topical application of atRA.

Correspondence to K. Yoshimura, M.D., Department of Plastic and Reconstructive Surgery, University of Tokyo, 7-3-1, Hongo, Bunkyo-Ku, Tokyo 113-8655, Japan
Tristimulus colorimeters and full-range or narrowband spectrophotometers have been employed for the quantification of erythema and pigmentation induced by ultraviolet radiation (UVR) [7], and for the color analysis of skin lesions [9] or transferred skin [12]. We have used a tristimulus colorimeter and a narrow-band spectrophotometer to evaluate skin reactions such as the intensity of pigmentation and erythema in our bleaching treatment. Representative cases are demonstrated and the usefulness of the reflectance spectrophotometer is discussed.

\section{Methods}

Combined topical applications of atRA, hydroquinone, and lactic acid were used for skin lesions with hyperpigmentation. atRA aqueous gel (atRA gel; 0.1, 0.2, and $0.4 \%$ ) was originally prepared at the Department of Pharmacy, University of Tokyo. AtRA gel was topically applied together with 5\% hydroquinone, 7\% lactic acid ointment (HQ-LA ointment), also prepared as described above. Plastibase (petrolatum polyethylene ointment base; Taisho Pharmacology, Osaka, Japan) was used as the ointment base of HQ-LA ointment. Both atRA gel and HQ-LA ointments are pharmacologically unstable, so that fresh ointments were prepared at least once a month. Each ointment was topically applied under signed informed consent to more than 120 Oriental patients with hyperpigmented skin lesions such as sinile lentigines, melasma, postinflammatory hyperpigmentation, and nevus spilus.

Treatment protocol. atRA gel was applied to the skin lesions twice a day, followed by the application of HQLA ointment. The concentration of atRA gel was changed according to the treated site: $0.1 \%$ atRA gel for the face, $0.2 \%$ for the trunk and upper extremities, and $0.4 \%$ for the lower extremities. In the daytime, a broad- 

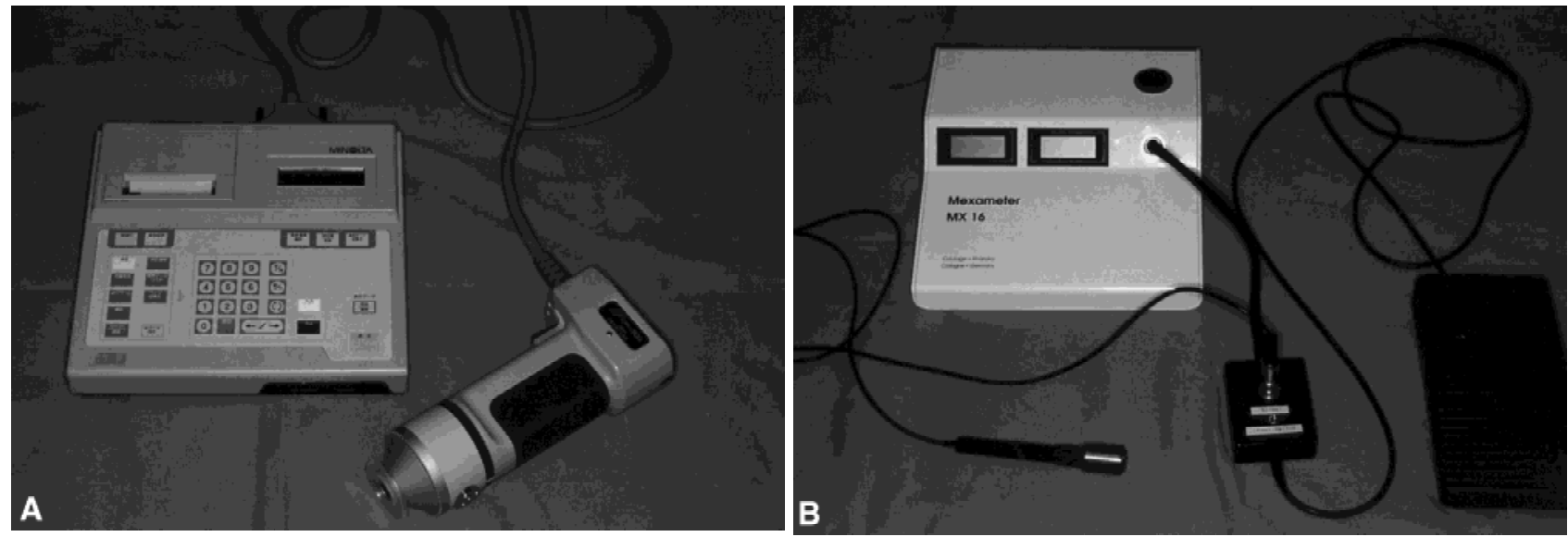

Fig. 1. (A) The tristimulus colorimeter (Chroma Meter CR-300). The control unit has a small printing unit and is connected to a measuring probe. This instrument offers five color systems for the data display including the CIE $L^{*} a^{*} b^{*}$ system. (B) The narrow-band reflectance spectrophotometer (Mexameter MX-16). The foot-switch model enables measurement without applying pressure to the skin. The melanin and hemoglobin values appear in the display of the control unit.

spectrum sunscreen cream was concomitantly applied throughout the treatment period. After improvement of the hyperpigmentation was obtained, the application of atRA was discontinued, and topical application of corticosteroids $(0.12 \%$ dexamethasone ointment) for 1 to 4 weeks was started to reduce the reactive erythema and inflammation. Throughout the therapy, topical application of HQ-LA ointment was continued except for cases in which erythema was not reduced after a few weeks' application of corticosteroid and HQ-LA ointment.

Measurement of skin color. As an objective measurement of the color of the designated lesion and normal skin, two types of portable reflectance instruments, a tristimulus colorimeter and a narrow-band reflectance spectrophotometer, were used at each clinical visit. During both measurements the photoreceivers were placed perpendicularly on the skin with minimal pressure. Each spot was measured three times and the average of the three measured values was calculated.

Instrumentation. Chroma Meter CR-300 (Minolta, Osaka, Japan) was used for colorimetry. $L^{*}, a^{*}$, and $b^{*}$ values of the CIE (Commission Internationale de l'Eclairage) system were measured. A color is expressed in a three-dimensional coordinate system with an $a^{*}$ axis (green-red), a $b^{*}$ axis (yellow-blue), and an $L^{*}$ axis (white-black) (see Refs. 1 and 4 for details). The $L^{*}$ value gives the relative brightness (or luminance), ranging from total black $\left(L^{*}=0\right)$ to total white $\left(L^{*}=100\right)$. $a^{*}$ is the component of separation between red (positive value) and green (negative value). $b^{*}$ represents the balance between yellow (positive) and blue (negative). The instrument consists of a control unit and a measuring probe for illuminating an area $8 \mathrm{~mm}$ in diameter (Fig. 1A). The measuring probe has a pulsed xenon lamp that emits an intense white light covering the entire visible spectrum (Chroma meter CR-300 instruction manual). The color of the reflected light is analyzed by three high- sensitivity silicone photocells that are filtered to match the CIE standard observer curves for the primary colors: blue $(450 \mathrm{~nm})$, green $(550 \mathrm{~nm})$, and red $(610 \mathrm{~nm})$.

The Mexameter MX 16 (Courage+Khazaka Electric $\mathrm{GmbH}$, Köln, Germany) was used for spectrophotometry. Because the original model employs a measuring probe that is placed on the spot to be measured with slight pressure, we ordered a foot-switch model with which measuring can be preformed without applying any pressure (Fig. 1B). A measuring probe with a measuring area of 5-mm diameter emits light of three predefined wavelengths $(568 \mathrm{~nm}$, green; $660 \mathrm{~nm}$, red; and $880 \mathrm{~nm}$, infrared) and measures the light reflected by the skin. The melanin value is measured using 2 wavelengths (660 and $880 \mathrm{~nm}$ ) to achieve different absorption rates by the melanin granules. For the hemoglobin measurement as well, two wavelengths (568 and $660 \mathrm{~nm}$ ) are used. The melanin and hemoglobin values are calculated as follows.

$\begin{aligned} \text { Melanin value }= & 500 / \log 5 \times[\log (\text { infrared-reflection } / \\ & \text { red-reflection })+\log 5]\end{aligned}$

Hemoglobin value $=500 / \log 5 \times[\log ($ red-reflection $/$ green-reflection) $+\log 5]$

Evaluation of treatment effects. The difference in the absolute melanin value between a skin lesion and normal skin is referred to as the relative melanin value (RMV) of the skin lesion in this paper, which indicates the intensity of pigmentation relative to the surrounding normal skin. RMV was calculated as

\section{$\mathrm{RMV}=\mathrm{AMV}($ lesion$)-\mathrm{AMV}($ normal $)$}

where AMV(lesion) and AMV(normal) are the absolute melanin values of a skin lesion and the surrounding normal skin, respectively.

In the same manner, the differences in the absolute hemoglobin values and the $L^{*}, a^{*}$, and $b^{*}$ values are 

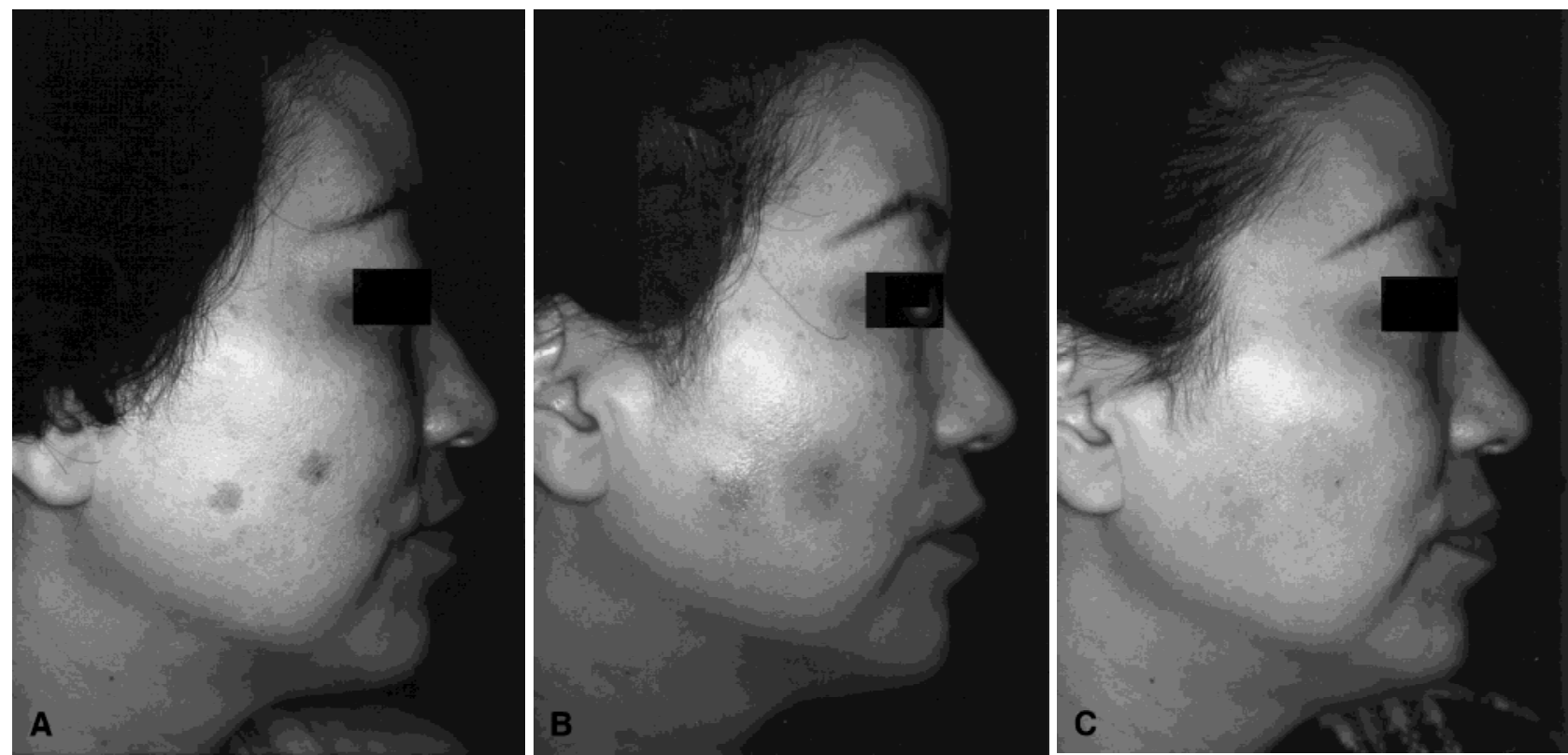

Fig. 2. (A) A pretreatment view of a 49-year-old woman with two senile lentigines on her right cheek. RMV $=47.7$, RHV $=24.3$, $(-) \Delta L^{*}=48.5, \Delta a^{*}=20.6, \Delta b^{*}=-1.5$. (B) After treatment for 3 weeks. Although moderate erythema was seen, pigmentation was reduced objectively and clinically. The topical application of atRA was discontinued. RMV $=-3.0, \mathrm{RHV}=89.3,(-) \Delta L^{*}=$ 38.6, $\Delta a^{*}=41.6, \Delta b^{*}=-12.1$. (C) After treatment for 10 weeks. The erythema was almost undetectable clinically. RMV $=-3.7$, $\mathrm{RHV}=33.3,(-) \Delta L^{*}=10.5, \Delta a^{*}=13.2, \Delta b^{*}=-11.9$.

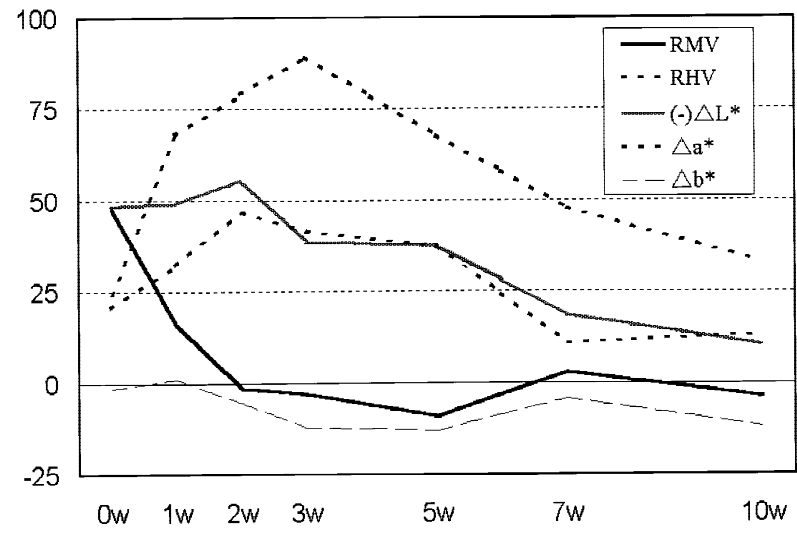

Fig. 3. Sequential changes in RMV, RHV, $(-) \Delta L^{*}, \Delta a^{*}$, and $\Delta b^{*}$ of case $1 .(-) \Delta L^{*}$ was plotted instead of $\Delta L^{*}$ for easier comparison to RMV.

designated RHV, $\Delta L^{*}, \Delta a^{*}$, and $\Delta b^{*}$, respectively, and calculated as described below:

$$
\begin{aligned}
\mathrm{RHV} & =\mathrm{AHV}(\text { lesion })-\mathrm{AHV}(\text { normal }) \\
\Delta L^{*} & =L^{*}(\text { lesion })-L^{*}(\text { normal }) \\
\Delta a^{*} & =a^{*}(\text { lesion })-a^{*}(\text { normal }) \\
\Delta b^{*} & =b^{*}(\text { lesion })-b^{*}(\text { normal })
\end{aligned}
$$

A negative RMV means that the measured spot is lighter than the control. The absolute melanin value of normal skin in Japanese and the RMV of hyperpigmented lesions are usually $460-500$ and $20-120$, respectively. A RMV of 5 or less is difficult to recognize clinically.

\section{Case Reports}

Case 1. A 49-year-old woman with two sinile lentigines on her right cheek underwent combined topical applications of $0.1 \%$ atRA gel and HQ-LA ointment (Fig. 2A: before treatment). The sequential changes in RMV, RHV, $\Delta L^{*}, \Delta a^{*}$, and $\Delta b^{*}$ are demonstrated in Fig. 3. On day 7 scaling was seen, and RMV was considerably reduced after the first 2 weeks, resulting in a negative RMV, while RHV was increased. Since an improvement of pigmentation was recognized objectively and clinically, atRA gel was discontinued despite the moderate erythema in the treated region (Fig. 2B: at 3 weeks). Corticosteroid was then applied topically for 4 weeks. Meanwhile, RHV gradually decreased and RMV remained around 0 . Three weeks after the discontinuation of corticosteroid treatment, the erythema disappeared almost completely and RHV was reduced almost to the level before treatment (Fig. 2C: at 10 weeks). HQ-LA ointment was applied throughout the treatment for 10 weeks. The RMV before treatment was 47.7 , and the final RMV after 10 weeks of treatment was -3.7. $\Delta a^{*}$ changed very similarly to RHV, while $\Delta b^{*}$ did not change significantly throughout the treatment. (-) $\Delta L^{*}$, which is the negative value of $\Delta L^{*}$, was slightly elevated during the first 2 weeks and then gradually decreased. 

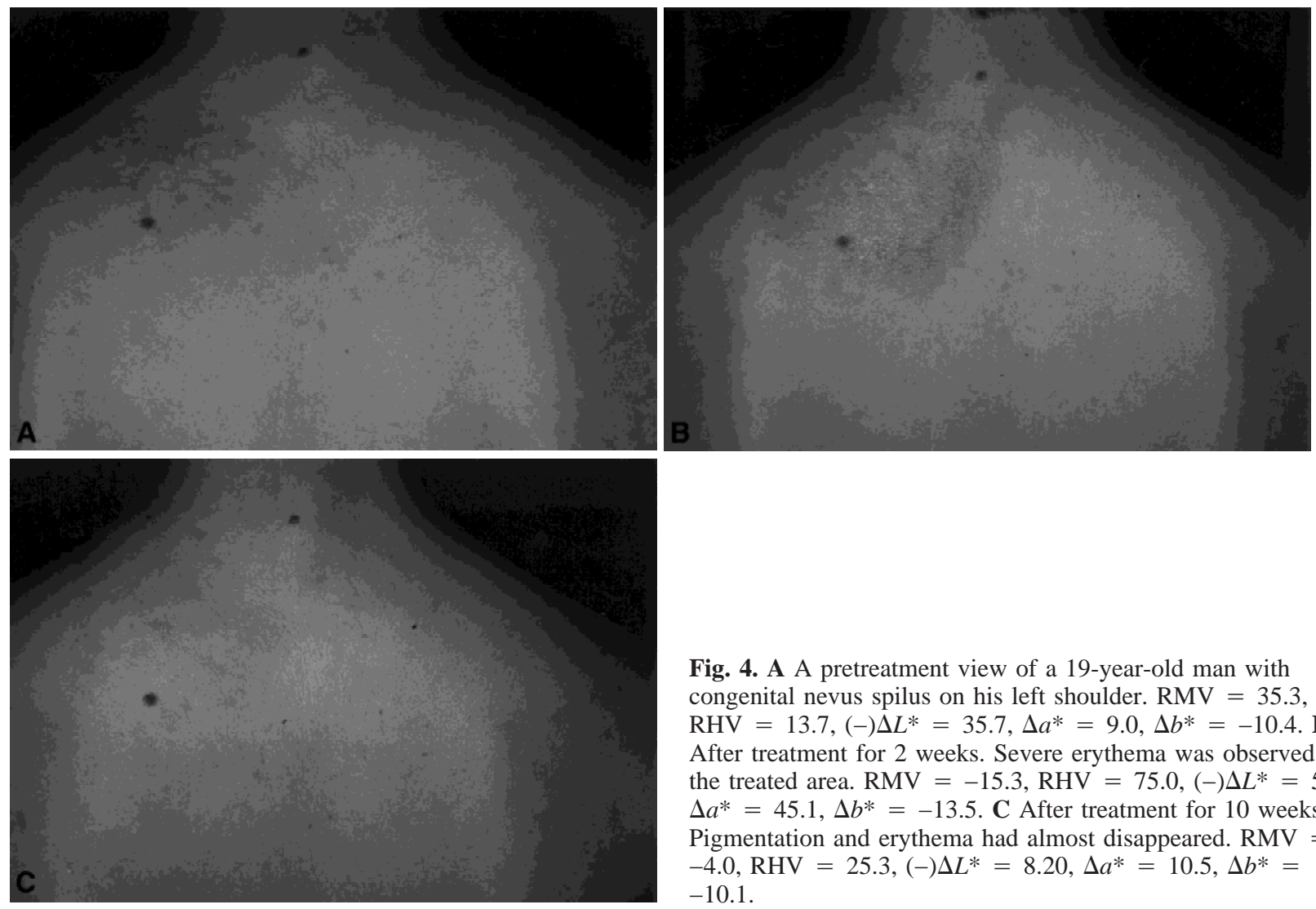

Fig. 4. A A pretreatment view of a 19-year-old man with congenital nevus spilus on his left shoulder. RMV $=35.3$, $\mathrm{RHV}=13.7,(-) \Delta L^{*}=35.7, \Delta a^{*}=9.0, \Delta b^{*}=-10.4 . \mathbf{B}$ After treatment for 2 weeks. Severe erythema was observed in the treated area. RMV $=-15.3, \mathrm{RHV}=75.0,(-) \Delta L^{*}=53.5$, $\Delta a^{*}=45.1, \Delta b^{*}=-13.5$. C After treatment for 10 weeks. Pigmentation and erythema had almost disappeared. RMV = $-4.0, \mathrm{RHV}=25.3,(-) \Delta L^{*}=8.20, \Delta a^{*}=10.5, \Delta b^{*}=$ -10.1 .

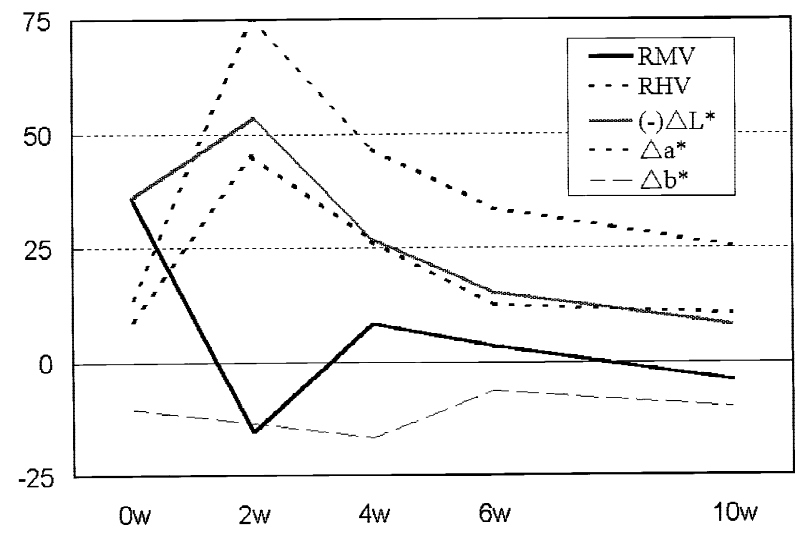

Fig. 5. Sequential changes in RMV, RHV, $(-) \Delta L^{*}, \Delta a^{*}$, and $\Delta b^{*}$ of case 2.

Case 2. A 19-year-old man with congenital nevus spilus on his left shoulder underwent combined topical applications of $0.2 \%$ atRA gel and HQ-LA ointment (Fig. 4A: before treatment). The sequential changes in RMV, RHV, $\Delta L^{*}, \Delta a^{*}$, and $\Delta b^{*}$ are demonstrated in Fig. 5. On day 5 scaling was seen, and at 2 weeks atRA gel was discontinued because RMV was reduced to a negative value. At that time, RHV was elevated to 75 and erythema was severe, so that it was not easy to estimate the extent of improvement of the pigmentation (Fig. 4B: at 2 weeks). HQ-LA ointment and corticosteroid were then applied for 3 weeks, followed by HQ-LA ointment application alone. Meanwhile, RHV was reduced gradually, while RMV was slightly elevated, presumably because of the temporary postinflammatory hyperpigmentation. At 5 weeks after the discontinuation of corticosteroid, the erythema had almost disappeared (Fig. 4C: after 10 weeks). The RMV before treatment was 35.3 , and the final RMV after 10 weeks of treatment was -4.0 . The sequential change in $\Delta a^{*}$ was quite similar to that of RHV, and $\Delta b^{*}$ was constant throughout the treatment period. $(-) \Delta L^{*}$ increased a little during the first 2 weeks and gradually decreased after 2 weeks.

\section{Discussion}

We have been successfully using high concentrations of atRA aqueous gel combined with hydroquinone and lactic acid as a depigmenting treatment for hyperpigmented skin lesions. In our protocol, aggressive retinoid treatment followed by the suppression of erythema and inflammation with corticosteroid can considerably shorten the treatment time and lead to satisfactory clinical results [14].

In this protocol, after a few weeks of treatment with atRA gel and HQ-LA ointment, hyperpigmentation was markedly reduced, frequently even resulting in a negative value of RMV. However, as shown in Figs. $2 \mathrm{~B}$ and $4 \mathrm{~B}$, moderate to severe erythema was usually observed in 
the treated areas. It is hard to evaluate clinically the improvement of pigmentation with accuracy when the lesion is overlaid with erythema. It is quite important in our depigmenting protocol to measure the intensity of pigmentation and erythema, not only to estimate the effect of the treatment but also to determine when atRA gel application should be discontinued and corticosteroid should be started. Although typical clinical time courses are demonstrated in Figs. $2 \mathrm{~A}-\mathrm{C}$ and $4 \mathrm{~A}-\mathrm{C}$, it may be necessary to modify the protocol in cases in which the pigmentation is not improved adequately after a few weeks of atRA treatment. The objective measurement of pigmentation and erythema was invariably a great help in deciding the treatment appropriate to the individual clinical situation.

In normal skin, the $a^{*}$ value showed a strong linear correlation with the erythema index of the spectrophotometer [11]. Similarly, $a^{*}$ showed a sequential change approximately parallel to that of RHV in our treatment (Figs. 3 and 5).

Although Seitz and Whitmore [10] suggested that the $b^{*}$ value was a good indicator of tanning, a recent study [7] disputed the correlation between the $b^{*}$ value and the melanin index. In Figs. 3 and 5, $\Delta b^{*}$ showed a small change during our treatment, and it is suggested that the $b^{*}$ value is not greatly affected by either pigmentation or erythema.

In a number of previous studies $[2,3,5,6,8]$, the $L^{*}$ value has been utilized as an index of skin pigmentation. However, there was only a weak correlation between the $L^{*}$ value and the melanin index detected in normal skin [7]. For easier comparison between the sequential changes in RMV and $\Delta L^{*}$ during our treatment, $(-) \Delta L^{*}$ was plotted in this paper. Figures 3 and 5 suggest that $(-) \Delta L^{*}$ was affected by both pigmentation and erythema. After a few weeks of treatment, although RMV was markedly reduced and the pigmentation was clinically reduced, $(-) \Delta L^{*}$ did not decrease or, rather frequently, increased. (-) $\Delta L^{*}$ as well as $\Delta a^{*}$ was elevated when erythema progressed, although RMV was reduced and appeared to keep indicating the intensity of pigmentation. It is therefore concluded that the $L^{*}$ value is not an appropriate index for pigmentation, especially when erythema is involved during treatments such as ours.

Although the CIE $L^{*} a^{*} b^{*}$ system has been widely used in a number of fields, it is hard to estimate the intensity of pigmentation with $L^{*}, a^{*}$, and $b^{*}$ values, but the narrow-band spectrophotometer was quite useful for that purpose, even when the pigmented lesions were overlaid with erythema.

\section{Conclusion}

Combined topical applications of all-trans retinoic acid aqueous gel and 5\% hydroquinone, 7\% lactic acid ointment have been successfully used as a depigmenting treatment for hyperpigmented skin lesions such as senile lentigines and nevus spilus. Although it was hard to translate the $L^{*}, a^{*}$, and $b^{*}$ values measured with a colorimeter to the intensity of pigmentation, a narrow-band reflectance spectrophotometer that measures the melanin and hemoglobin indices was found to be quite useful for estimating accurately the intensity of pigmentation and erythema and determining when the application of atRA treatment should be discontinued.

Acknowledgments. We express our sincere appreciation to Yuka Kuwahara and Takako Kato for their assistance in colorimetric measurement.

\section{References}

1. Andreassi L, Flori L: Practical applications of cutaneous colorimetry. Clin Dermatol 13:369, 1995

2. Burns RL, Prevost-Bank PL, Lawry MA, Lawry TB, Faria DT, Fivenson DP: Glycolic acid peels for postinflammatory hyperpigmentation in black patients. Dermatol Surg 23:171, 1997

3. Duteil L, Ortonne JP: Colorimetric assessment of the effects of azelaic acid on light-induced skin pigmentation. Photodermatol Photoimmunol Photomed 9:67, 1992

4. Fullerton A, Fischer T, Lahti A, Wilhelm KP, Takiwaki H, Serup J: Guidelines for measurement of skin colour and erythema. Contact Dermatitis 35:1, 1996

5. Griffiths CEM, Goldfarb MT, Finkel LJ, Roulia V, Bonawitz M, Hamilton TA, Ellis CN, Voorhees JJ: Topical tretinoin (retinoic acid) treatment of hyperpigmented lesions associated with photoaging in Chinese and Japanese patients: A vehicle-controlled trial. J Am Acad Dermatol 30:76, 1994

6. Kimbrough-Green CK, Griffiths CEM, Finkel LJ, Hamilton TA, Bulengo-Ransby SM, Ellis CN, Voorhees JJ: Topical retinoic acid (tretinoin) for melasma in black patients. Arch Dermatol 130:727, 1994

7. Kollias N, Baqer AH: Quantitative assessment of UVinduced pigmentation and erythema. Photodermatology 5: 53, 1988

8. Maeda M, Kachi H, Matubara K, Mori S, Kitajima Y: Pigmentation abnormalities in systemic scleroderma examined by using a colorimeter. J Dermatol Sci 11:228, 1996

9. Marchesini R, Brambilla M, Clemente C, Maniezzo M, Sichirollo AE, Testori A, Venturoli DR: In vivo spectrophotometric evaluation of neoplastic and non-neoplastic skin pigmented lesions-I. Reflectance measurements. Photochem Photobiol 53:77, 1991

10. Seitz JC, Whitmore CG: Measurements of erythema and tanning responses in human skin using a tristimulus colorimeter. Dermatologica 177:70, 1988

11. Takiwaki H, Overgaard L, Serup J: Comparison of narrowband reflectance spectrophotometric and tristimulus colorimetric measurements of skin color. Skin Pharmacol 7:217, 1994

12. Yamamoto Y: Colorimetric evaluation of skin color in the Japanese. Plast Reconstr Surg 96:139, 1995

13. Yoshimura K, Harii K: A new treatment for senile lentigines. J Jpn Plast Reconstr Surg 17:630, 1997

14. Yoshimura K, Harii K, Aoyama T, Shibuya F, Iga T: A new bleaching protocol for hyperpigmented skin lesions with a high concentration of all-trans retinoic acid aqueous gel. Aesth Plast Surg (in press) 